\title{
Bursty Traffic Modelling and Multiplexing Performance Analysis in ATM Networks: A Three-moment Approach*
}

\author{
N. Mitrou(a), K. Kontovasilis(a), and V. Nellas(a)
}

(a) National Technical University of Athens, Dept. of Electrical and Computer Eng., Heroon Polytechneiou 9, GR-15773, Zografou, Greece

\begin{abstract}
Modelling of hyper-exponential ON/OFF traffic streams by a four-state Markov Modulated Rate Process (MMRP) is considered as a means of obtaining a sufficiently accurate, yet computationally tractable analytical model for ATM traffic. A general formulation of the proposed model along with the associated multiplexing analysis is first developed. Then, a special time-reversible version of the model that arises naturally in many practical configurations of interest is analysed, which enables the modelling and the parameter assessment of the $O N$ and $O F F$ phases independently as two-stage, Erlang-type processes. Application examples with comparison of analysis, simulation and experimental results derived on an ATM testbed are given.
\end{abstract}

\section{INTRODUCTION}

Traffic modelling and the related performance analysis is a crucial step towards the ability of devising sensible dimensioning rules and flexible algorithms for traffic control in communication networks. These issues become even more important to an ATMnetworking environment, due to the multiplexing of bursty traffic streams, of diverse profiles, over the same network components (multiplexers, switches) [17]. Balancing the inherent trade-off between simplicity and accuracy of the potential models is always a critical point of the modelling procedure.

A class of models that offers both sufficient flexibility and reasonable analytical tractability belong to the so-called Markov Modulated Rate Processes (MMRP) [11],[1],[19]. The models of this class ignore the discrete nature of the ATM traffic (actually conveyed by discrete cells), considering instead a continuous flow of information. Such an approximation is quite reasonable, since in a well-dimensioned system the buffer at the multiplexers should be large enough to absorb cell-level congestion (i.e. congestion due to simultaneous cell arrivals). The rate of information flow in an MMRP is assumed to be modulated by an underlying Markov chain, being kept constant within each individual state of the chain. The simplest member of this class is the one-state model, resulting in Constant Bit Rate (CBR) streams. The bandwidth allocation and connection admission procedures are trivial in that

\footnotetext{
* This work was partly done within the frame of the RACE EXPLOIT project
} 
case (peak rate allocation). However, allocating the peak rate to Variable Bit Rate (VBR) connections may lead to a substantial waste of bandwidth, because the potential multiplexing gain is not exploited.

A two-state MMRP, alternating between a low-rate state (usually zero-rate, OFF) and a high-rate state $(\mathrm{ON})$, is the simplest model that renders a burstiness (peak-over-mean-rate ratio) greater than unity and allows for a statistical multiplexing gain to be exploited. A great deal of attention has been drawn upon that model. This effort is motivated by the ability of this model to describe bursty traffic and the tractability of the related multiplexing analysis [1],[11]. A single parameter descriptor, the Effective Rate (ER), can be formulated and calculated rather easily [7],[6],[15], while traffic shaping for ER enforcement is also possible [14],[15]. Variable-Bit-Rate (VBR) traffic, like VBR video, can in some cases be modelled as a superposition of simple ON/OFF traffic streams [12].

The ON/OFF type of traffic seems to be of the most frequently encountered in the forthcoming ATM networks. By realising that VBR or multirate traffic streams are difficult to control and to get a profit out of their statistical multiplexing, transforming them into $\mathrm{ON} / \mathrm{OFF}$ streams, through suitable shaping at the transmitter, offers a promising approach to handling bursty traffic without sacrificing the multiplexing gain. However, the case of exponentially distributed $\mathrm{ON}$ and OFF periods, which is accurately modelled by a two-state MMRP, will only be an exception. Consider, for example, the case of a data-packet stream, transmitted (shaped) at a certain peak rate. Even when the original data packets are exponentially sized, the resulting cell stream exhibits a hyper-exponential $\mathrm{ON}$ duration, due to merging of consecutive packets [16] and, as will be shown, the variance of the ON and OFF sojourn times is critical for the multiplexer's performance. A hyper-exponential distribution, in particular (e.g. of the Erlang type, representable by parallel exponential stages), gives a higher Complementary Probability Distribution Function (CPDF) for the queue length in the long run and, therefore, worse performance in terms of both the delay and the cell loss probability, as compared to the exponential distribution with the same mean. In [20] it was found that the cell streams within the switching network can be modelled with hyper-exponential distributions for both ON and OFF periods. In such cases the simple exponential ON/OFF model fails to give acceptable results and models of a higher dimension are necessary.

In [16] a three-state MMRP is used to model ON/OFF traffic with hyper-exponentially distributed ON sojourn times, the parameters of which may be directly assessed by measurements on the real traffic stream. In the present paper, a more general, four-state model is used to describe ON/OFF streams, with emphasis on the parameter matching and the analytical solution of the statistical multiplexing problem. First, a four-state MMRP is set up and the general formulation of the related multiplexing problem is given in short (section 2). In the same section, a time-reversibility condition for the model at hand is given. In section 3, the solution of the generalised eigenvalue problem associated with the multiplexer's performance is elaborated via a well-established factorization method. Section 4 is devoted to the parameter assessment problem by using a reduced version of the model, which enables the modelling of the $\mathrm{ON}$ and OFF phases independently by an Erlang-type process, through matching their first three moments with real measurements. Application examples are given in section 5, along with comparison results between different low order MMRPs. Experimental results derived on a real ATM Testbed are also included. 
The basic assumption underlying the proposed three-moment-matching approach is that ON/OFF streams, resulting from shaping of data or VBR traffic, can be modelled so as ON and OFF sojourn times are mutually independent and each instance of them is independently drawn from specific distributions, different, in general, for each of the two phases. Such a simplification is reasonable, since the shaping process eliminates much of the correlation which possibly exists between the original information blocks produced by the traffic source. Additionally, it is assumed that the coefficient of variation of the $\mathrm{ON}$ and OFF sojourn times is greater than or equal to unity (hyper-exponential or exponential ONOFF phases). The hypo-exponential case results in a better performance than its exponential counterpart, thus it can be safely modelled by the latter.

\section{MMRP MODELLING AND FORMULATION OF THE MULTIPLEXING PROBLEM}

In this section the four-state Markov Modulated Rate Process, proposed for modelling $\mathrm{ON} / \mathrm{OFF}$ traffic streams, is established and the related multiplexing analysis problem is formulated. A necessary and sufficient condition for time reversibility is also given, which, when valid, facilitates the solution of the multiplexing problem.

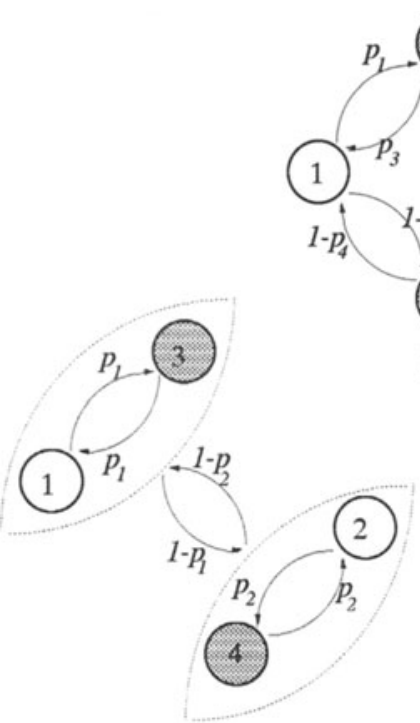

(b)

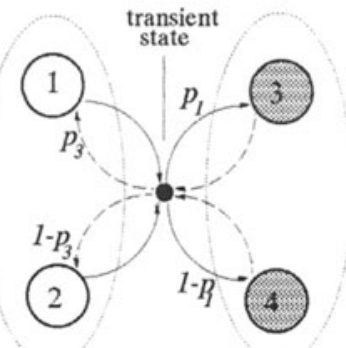

OFF phase
ON phase

(c)

Figure 1: Four-state MMRP modelling of ON-OFF streams (a) general hyperexponential model (b) case with $p_{1}=p_{3}, p_{2}=p_{4}$ (c) case with $p_{1}=1-p_{2}, p_{3}=1-p_{4}$ 
Consider the model of fig. 1(a). States 1 and 2 are the OFF states, at which no information is transmitted, while states 3 and 4 are the ON states, where information is transmitted at a constant rate, $c$. The sojourn time at each state, $j$, is assumed to be exponentially distributed, with a mean equal to $\tau_{j}, j=1,2,3,4$. Transition probabilities are shown on the corresponding arrows, which are assumed to take values different than zero or one. The infinitesimal generator of the Markov chain and the respective diagonal flow-rate matrix, that describe the rate process, are then expressed as

$$
M=\left[\begin{array}{cccc}
-\frac{1}{\tau_{1}} & 0 & \frac{p_{1}}{\tau_{1}} & \frac{1-p_{1}}{\tau_{1}} \\
0 & -\frac{1}{\tau_{2}} & \frac{1-p_{2}}{\tau_{2}} & \frac{p_{2}}{\tau_{2}} \\
\frac{p_{3}}{\tau_{3}} & \frac{1-p_{3}}{\tau_{3}} & -\frac{1}{\tau_{3}} & 0 \\
\frac{1-p_{4}}{\tau_{4}} & \frac{p_{4}}{\tau_{4}} & 0 & -\frac{1}{\tau_{4}}
\end{array}\right] \quad, R=\operatorname{diag}(0,0, c, c)
$$

In the rest of this subsection, the formulation of the multiplexing problem is reviewed in a fairly general setting, while a solution via a factorization method is exposed in section 3 .

Considering the superposition of $N$ independent streams of the above type, the composed traffic is still an MMRP with global matrices

$$
M_{g}=M^{(1)} \oplus M^{(2)} \oplus \ldots \oplus M^{(N)} \quad \text { and } \quad R_{g}=R^{(1)} \oplus R^{(2)} \oplus \ldots \oplus R^{(N)}
$$

respectively, where the superscript $(n)$ refers to the $n_{\text {th }}$ stream, $n=1,2, \ldots N$, and where $\oplus$ denotes the Kronecker sum operator. $A$ similar notation will also apply to all of the parameters relevant to the matrices $M^{(n)}$ and $R^{(n)}$ given by (1).

The state-space comprises $\aleph=4^{N}$ states. In the case of a homogeneous traffic mix, however, it can be compressed to $\left(\begin{array}{c}N+3 \\ 3\end{array}\right)$ states, by recognising the statistically identical feature of the superposed streams. This is possible because homogeneity allows to keep track of only the number of sources that are in a particular micro-state 1...4, instead of the micro-state of all the sources on an individual basis.

Define a vector

$\vec{F}(x)=\left(F_{1}(x), \ldots, F_{\aleph}(x)\right)$, where $F_{i}(x)=\operatorname{Pr}\{$ state is $i$ and buffer content $\leq x\}$. 
Then, at steady state, the system satisfies the differential equations

$$
\frac{d}{d x} \vec{F}(x) D=\vec{F}(x) M_{g},
$$

where $M_{g}$ is given by (2), $D=R_{g}-C I, C$ is the output link rate of the multiplexer and $I$ is the identity matrix of appropriate order. Solution of (3) yields $\vec{F}(x)$ that completely describes buffer occupancy.

When $M_{g}$ is time-reversible, then the generalised eigenvalue problem

$$
z \vec{\varphi} D=\vec{\varphi} M_{g}
$$

has a full set of left-eigenvectors and all respective eigenvalues are real (see e.g. appendix in [13]). A time-reversibility condition for the special models discussed here, along with special cases fulfilling that condition are given in the next subsection. Furthermore, each eigenvalue $z_{i}$ that corresponds to a state $i$ with respective flow rate $R_{g}(i, i)<C$ is non-negative and the respective state is said to be underload. Similarly, $z_{i}<0$, for all states $i$ with $R_{g}(i, i)>C$, the relevant states being called overload.

By denoting the set of overload states as $S_{o}=\left\{i \mid R_{g}(i, i)>C\right\}$, the vector $\vec{F}(x)$, for an infinite buffer system ${ }^{1}$, can be written as

$$
\vec{F}(x)=\vec{\pi}+\sum_{i \in S_{o}} \alpha_{i} \vec{\varphi}_{i} e^{z_{i} x},
$$

where the $\bar{\varphi}_{i}$ and $z_{i}$ are eigenvalue-eigenvector pairs of the system (4), $\vec{\pi}$ is the steady state vector of $M_{g}$ and $\alpha_{i}$ are appropriate coefficients derived by considering the boundary conditions.

By exploiting the Kronecker form in (2) as in [19] and [11], the computational burden associated with the solution of the eigenvalue problem (4) can be significantly reduced. A solution of this kind will be discussed in the following section 3. Additionally, in [19,8,2] there can be found methods for obtaining bounds to the coefficients $\alpha_{i}$, thus avoiding the burden of solving for the large system of linear equations.

In practice the vector $\vec{F}(x)$ is very detailed to be useful. The analyst's interest usually lies in the survivor function of the buffer occupancy $G(x)=1-\vec{F}(x) \cdot \overrightarrow{1}$ ( $\overrightarrow{1}$ is a column vector of all ones, of appropriate size). By using (5) one immediately realises that

\footnotetext{
1 A respective finite buffer formulation may be constructed, the only difference being in the enforcement of the boundary conditions. For the low probabilities encount, red in ATM studies, however, the difference between the infinite and finite buffer metrics is negligible and thus the infinite-buffer model is preferred on the basis of stabler computations. In any case, it provides conservative upper bounds to the "true" finite-case results.
} 


$$
G(x)=-\sum_{i \in S_{o}} \alpha_{i}\left(\vec{\varphi}_{i} \cdot \overrightarrow{1}\right) e^{z_{i} x} .
$$

Furthermore, and although in practice the buffer size is always finite, say $V_{b}$, the quantity $G\left(V_{b}\right)$, as derived by the infinite buffer model, is, at the low probabilities of buffer overflow that ATM multiplexers are engineered for, a close upper bound to the exact overflow probability of the finite buffer of size $V_{b}$. Thus, in all domains of interest, the easier to solve and more well conditioned infinite-buffer model can be used to yield results for multiplexers equipped with a finite buffer.

\section{Time-reversibility condition}

It is easy to show (Appendix A) that the necessary and sufficient condition for the matrix $M$ in (1) to be time-reversible is:

$$
\left(\frac{1-p_{1}}{p_{1}}\right)\left(\frac{1-p_{2}}{p_{2}}\right)=\left(\frac{1-p_{3}}{p_{3}}\right)\left(\frac{1-p_{4}}{p_{4}}\right)
$$

Moreover, if the matrices involved in the Kronecker product of (2) are time-reversible, the same holds true for the global matrix $M_{g}$ itself. Two important cases that obviously satisfy the time-reversibility condition (7) are:

$$
\text { Case I: } \quad p_{1}=p_{3}, p_{2}=p_{4} \text { or } p_{1}=p_{4}, p_{2}=p_{3}
$$

Case II: $\quad p_{1}=1-p_{2}, p_{3}=1-p_{4}$

In the first case each ON state can be combined with one OFF state to form a macrostate of the ON-OFF type, e.g. with $p_{1}=p_{3}, p_{2}=p_{4}$ we get the macro-states 1-3 and 2-4, as shown in fig. 1(b). If, in addition, $p_{1}>>1-p_{1} \quad \& \quad p_{2}>>1-p_{2}$, the two macro-states are loosely coupled (NCD structure [3]) allowing for approximate analysis procedures to apply [9],[10].

In case (II) the ON states are not correlated with the OFF states, in the sense that a transition to a certain ON state does not depend on the particular OFF state it originates from, and vice-versa. This is schematically shown in fig. 1(c), where a transient state with a zero sojourn time is used for the decoupling of the sort just described. This case has the distinct advantage of allowing the assessment of the parameters of the $\mathrm{ON}$ and OFF states separately, as will be exploited in section 4 . 


\section{SOLUTION VIA DECOMPOSITION}

Solving the generalised eigenvalue problem (4) by brute-force techniques possesses severe difficulties, even for moderately large values of $N$. In [19] a factorization method is proposed which exploits the Kronecker product form of (2) to solve (4) in a computationally efficient way. Application of this method here leads to an analytical solution, except for a simple Newton-Raphson procedure when determining the eigenvalues of the global system.

Following the formalism of [19], for each multiplexed stream with matrices $M^{(n)}$ and $R^{(n)}$ given by (1) we form the matrix

$$
A^{(n)}(z)=R^{(n)}-M^{(n)} / z
$$

and solve for its four eigenvalues, namely $g^{(n)}(j ; z), j=1,2,3,4$, which are functions of $z$. In Appendix B, the functions $g($.$) are computed in closed form as the four roots of a$ polynomial equation

$$
g^{4}+\beta_{1} g^{3}+\beta_{2} g^{2}+\beta_{3} g+\beta_{4}=0
$$

where the coefficients $\beta_{j}, j=1,2,3,4$, depend on the model parameters and the value of $z$.

By assuming that the time-reversibility condition (7) is satisfied, $A^{(n)}(z)$ has a full set of independent left eigenvectors and all four eigenvalues (roots of (10)) are real for any value of $z$. The asymptotic behaviour of $g^{(n)}(j ; z)$ (see [19]) can be summarised as

$$
\begin{array}{ll}
\lim _{z \rightarrow 0+} g^{(n)}(1 ; z)=\lim _{z \rightarrow 0-} g^{(n)}(4 ; z)=r^{(n)} \\
\lim _{z \rightarrow 0+} g^{(n)}(j ; z)=+\infty, j=2,3,4 & \lim _{z \rightarrow 0-} g^{(n)}(j ; z)=-\infty, j=1,2,3 \\
\lim _{z \rightarrow \pm \infty} g^{(n)}(j ; z)=0, j=1,2 & \lim _{z \rightarrow \pm \infty} g^{(n)}(j ; z)=c^{(n)}, j=3,4
\end{array}
$$

where $r^{(n)}$ is the average rate of the $n_{\text {th }}$ stream at hand, equal to $c^{(n)}\left(\pi_{3}^{(n)}+\pi_{4}^{(n)}\right)$, where $\pi_{3}^{(n)}, \pi_{4}^{(n)}$ denote the steady-state probabilities of the ON states, 3,4 respectively. Typical curves of the $g^{(n)}(j ; z)$ for negative values of $z$ are shown in fig. 2 . They correspond to the model (vii) of Example 1. 


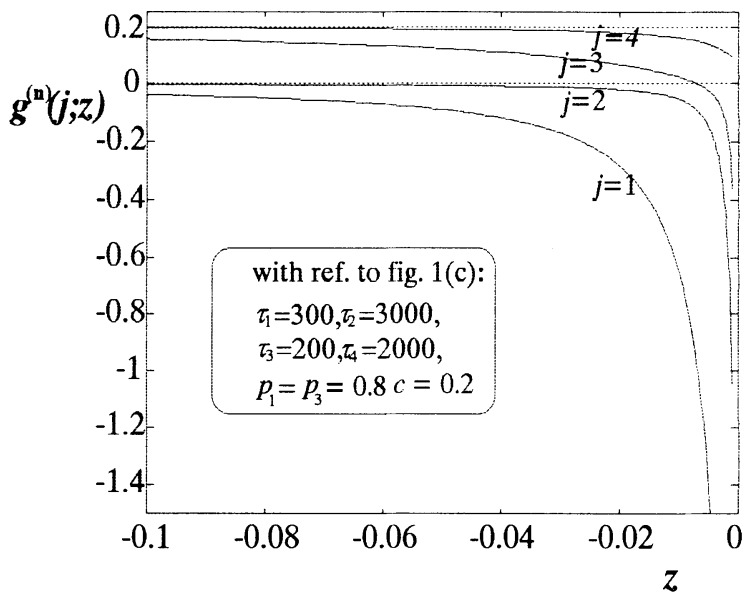

Figure 2: Typical $g^{(n)}(j ; z)$ curves

Consider now the multiplexing of $N$ independent, non-identical such streams. The global system is characterised by the matrices $M_{g}$ and $R_{g}$ given by (2). As already mentioned earlier, the time-reversibility condition (7) holding true for all individual streams implies time-reversibility for the global system too. The negative eigenvalues of the global system correspond one-to-one to its overload states, the subspace of which can be written as

$$
S_{o}=\left\{i \leftrightarrow\left(j^{(1)}, j^{(2)}, \ldots, j^{(N)}\right): \sum_{\substack{n=1 \\ j^{(n)} \in\{3,4\}}}^{N} c^{(n)}>C\right\},
$$

where $j^{(n)} \in\{1,2,3,4\}$ the state of the $n_{\text {th }}$ stream, and $c^{(n)}$ its peak rate (for $j^{(n)} \in\{3,4\}$ ). The symbol $\leftrightarrow$ in (12) associates a state number, $i$, with its state vector according to a lexicographic ordering scheme.

For any $i \in S_{o}$, the respective (negative) eigenvalue, $z_{i}$ can be uniquely determined by solving the equation

$$
\sum_{n=1}^{N} g\left(j^{(n)} ; z_{i}\right)=C,
$$

The respective eigenvector is given by the Kronecker product

$$
\vec{\varphi}_{i}=\vec{\varphi}_{i}^{(1)} \otimes \vec{\varphi}_{i}^{(2)} \otimes \ldots \otimes \vec{\varphi}_{i}^{(N)}
$$


where $\bar{\varphi}_{i}$ denotes the left eigenvector of the $A^{(n)}(z)$, associated with the respective eigenvalue, $g^{(n)}\left(j^{(n)} ; z_{i}\right)$.

To this end, all the computations are performed in closed form, except equation (13) which must be solved numerically. Since the functions $g($.$) are well-behaved, a simple$ second-order iterative technique such as the Newton-Raphson's method is applicable. The derivative of the $g$ 's required by such a method is also given in closed form (see Appendix B).

\section{Aggregation of states in the homogeneous traffic case}

As already mentioned, superposition of $N$ 4-state streams results, due to the Kronecker sum form of (2), in a global state-space of size $4^{N}$. If the superposed streams are statistically homogeneous, many of the $4^{N}$ states are statistically equivalent. Thus an equivalent model

of only $\left(\begin{array}{c}N+3 \\ 3\end{array}\right)$ states may be constructed, reducing the state explosion from an exponential complexity down to $\mathrm{O}\left(N^{3}\right)$.

The state description from an $N$-long vector $\left(j^{(1)}, j^{(2)}, \ldots, j^{(N)}\right), 1 \leq j^{(n)} \leq 4$ (as it appears in (12)), expressing the particular state visited by each of the $N$ streams, may be reduced to

a 4-long vector $\left(m_{1}, m_{2}, m_{3}, m_{4}\right)$ with $\sum_{j=1}^{4} m_{j}=N$, retaining only the multiplicities with which the 4-states occur in the $N$ streams. Thus the overload subspace in the new representation becomes

$$
S_{o}=\left\{i \leftrightarrow\left(m_{1}, m_{2}, m_{3}, m_{4}\right), \sum_{j} m_{j}=N: c\left(m_{3}+m_{4}\right)>C\right\}
$$

and, similarly, the equation to be solved for the $i$-th eigenvalue

$$
\sum_{j=1}^{4} m_{j} g\left(j ; z_{i}\right)=C,
$$

with the functions $g($.$) as before. Note that in this subsection we have dropped the$ superscript identifying individual streams, since homogeneous multiplexing is considered.

However, the eigenvectors as given by (14) are in the unaggregated form. To obtain the compressed counterparts we need to sum together all the equivalent (unaggregated) states. An efficient algorithm that performs this eigenvector aggregation, discussed in [5], may be applied. This algorithm takes as input the 4 "partial" eigenvectors of $A\left(z_{i}\right)$ corresponding to $g\left(j ; z_{i}\right), j=1, \ldots, 4$, and the multiplicities $m_{j}, j=1, \ldots, 4$, with which these four states occur in the $N$ multiplexed streams and produces the aggregated eigenvector. It is obvious that when heterogeneous classes, each including several statistically identical streams, are 
multiplexed together, the discussion above applies to each class separately. This leads to a state space of $\prod_{k=1}^{K}\left(\begin{array}{c}N_{k}+3 \\ 3\end{array}\right)$ states, when $K$ classes of $N_{k}$ streams, $k=1,2, \ldots K, \quad$ are multiplexed together.

\section{PARAMETER ASSESSMENT}

We turn now to an important issue, usually attracting little attention in the literature, namely that of assessing the model parameters in terms of real measurements. The approach to this problem, followed here, is based on matching the first three moments of the $\mathrm{ON}$ and $\mathrm{OFF}$ sojourn times (composite states 3-4 and 1-2, respectively) independently for each phase, with those of the real stream, as proposed in [16]. To this end, we assume that the model of fig. 1(c) is applicable, where the transition to a certain ON (OFF) state is independent of the particular OFF (ON) state it originates from. This memoryless property is modelled by using a virtual transient state to decouple the ON and OFF phases as shown in fig. 1(c). This decoupling is a reasonable compromise, since it is very costly to gather information on the coupling the ON and OFF phases from measured data. The model at hand is a special case of the more general model of fig. 1(a) with $p_{1}=1-p_{2}, p_{3}=1-p_{4}$ and is time-reversible, as mentioned earlier. The moments are measured by collecting samples of talkspurt and silence durations as follows:

During call-setup, the source declares its peak rate $c$, together with a 'maximum' celldelay variation $\Delta$. Based on these figures, the maximum allowable distance between successive cells during a talkspurt would be $1 / c+\Delta$. Thus, after a cell has been received, the sampler waits for $1 / c+\Delta$ slots. If, during that interval another cell arrives, then the talkspurt is assumed to be continued (and this timing is reinstated from the new arrival slot). If no cell arrives during the timing, it is assumed that the talkspurt has ended and a silence phase begins when the the timing has expired. A cell arrival during a silence phase of course terminates this silence and begins a new talkspurt. Note that contributing the timing interval in the talkspurt, rather than the silence results in a model with slightly larger talkspurts and slightly smaller silences. This is a conservative approach that yields a model with worse performance, keeping the performance prediction on the safe side.

Of course, there is always the possibility that two successive cells within a talkspurt will be spaced apart a bit more than $1 / c+\Delta$. In this case, the sampling algorithm will be fooled to think that a 'big' talkspurt is two 'small' talkspurts at both sides of a 'small' silence. This tends to give small smaller values for the moments of both talkspurt and silence (because samples smaller than the actual ones are assumed). However, since this affects both talkspurts and silences the measurement errors are somewhat counterbalanced.

Denote by $t_{1}, t_{2}, t_{3} \& s_{1}, s_{2}, s_{3}$ the first three non-central moments of the ON and OFF phases, respectively. As shown in [16] (see also Appendix C), the model parameters are then expressed as 


$$
\begin{array}{lll}
\tau_{1}=\frac{x_{s}-\sqrt{x_{s}^{2}-4 y_{s}}}{2}, & \tau_{2}=\frac{x_{s}+\sqrt{x_{s}^{2}-4 y_{s}}}{2}, & p_{1}=\frac{\tau_{2}-s_{1}}{\tau_{2}-\tau_{1}} \\
\tau_{3}=\frac{x_{t}-\sqrt{x_{t}^{2}-4 y_{t}}}{2}, & \tau_{4}=\frac{x_{t}+\sqrt{x_{t}{ }^{2}-4 y_{t}}}{2}, & p_{3}=\frac{\tau_{4}-t_{1}}{\tau_{4}-\tau_{3}}
\end{array},
$$

where

$$
x_{s}=\frac{\frac{s_{3}}{6}-\frac{s_{2}}{2} s_{1}}{\frac{s_{2}}{2}-s_{1}^{2}}, y_{s}=s_{1} x_{s}-\frac{s_{2}}{2}, x_{t}=\frac{\frac{t_{3}}{6}-\frac{t_{2}}{2} t_{1}}{\frac{t_{2}}{2}-t_{1}^{2}}, y_{t}=t_{1} x_{t}-\frac{t_{2}}{2}
$$

Obviously, the model of fig. 1(c) renders a hyper-exponential distribution (Erlang-type with two stages in parallel) for both phases, ON and OFF. Consequently, in order for the above matching to be possible, the three moments of each phase must fulfil a hyperexponentiallity condition, i.e.

$$
\begin{array}{ll}
t_{2}>2 t_{1}{ }^{2}, & t_{3}>\frac{3 t_{2}{ }^{2}}{2 t_{1}} \\
s_{2}>2 s_{1}{ }^{2}, & s_{3}>\frac{3 s_{2}{ }^{2}}{2 s_{1}}
\end{array}
$$

Condition (16) is necessary and sufficient (see Appendix C) to make all quantities in (15) positive, as expected.

The extreme case of $\left[t_{2}=2 t_{1}{ }^{2}, t_{3}=6 t_{1}{ }^{3}\right]$ or / and $\left[s_{2}=2 s_{1}{ }^{2}, s_{3}=6 s_{1}{ }^{3}\right]$ makes the corresponding phase(s) exponential, i.e. $\tau_{1}=\tau_{2}$ or $/$ and $\tau_{3}=\tau_{4}$, thus reducing the model to a three- or a two-state MMRP. Such a model is much easier to handle (see [16], [1]) as is briefly discussed in the subsection to follow. A comparison of the multiplexing analysis results between all these models is performed in the last section (Example 1).

When the inequalities in (16) are in the reverse direction, the corresponding phase(s) is (are) hypo-exponential. These cases cannot be handled by the proposed model. However, it has been observed that a hypo-exponential distribution of the $\mathrm{ON}$ and/or the OFF phase gives a better performance (i.e. a lower buffer occupancy distribution) than the equivalent exponential or hyper-exponential process with the same mean values (see Example 1). Hence we are on the safe side (more pessimistic) if we model a hypo-exponential phase as an exponential one, matching only the first moment. 


\section{Reduction to a three-state model}

When $\tau_{1}=\tau_{2}\left(\tau_{3}=\tau_{4}\right)$ then the corresponding $\mathrm{OFF}(\mathrm{ON})$ phase becomes exponential and the model is reduced to a three-state one. Let us examine the case of $\tau_{1}=\tau_{2}$, corresponding to an exponential OFF phase. The infinitesimal generator of the Markov chain and the flow-rate matrix of the reduced model are given by

$$
M=\left[\begin{array}{ccc}
-\frac{1}{\tau_{1}} & \frac{p_{1}}{\tau_{1}} & \frac{1-p_{1}}{\tau_{1}} \\
\frac{1}{\tau_{3}} & -\frac{1}{\tau_{3}} & 0 \\
\frac{1}{\tau_{4}} & 0 & -\frac{1}{\tau_{4}}
\end{array}\right], R=\operatorname{diag}(0, c, c)
$$

Such a model is always time reversible, as it can be easily verified and the three $g($. functions (i.e. the eigenvalues of the matrix $A(z)=R-M / z$ ) are the roots of a polynomial equation

$$
g^{3}+\delta_{1} g^{2}+\delta_{2} g+\delta_{3}
$$

The coefficients $\delta_{j}, j=1,2,3$, and the solution of (18) are given for the sake of completeness in Appendix B. In [16] a more detailed study of this model is presented, with emphasis on its NCD structure, resulting from the condition $p_{1}>1-p_{1}, \tau_{4}>\tau_{3}$.

\section{EXAMPLES}

\section{Example 1:}

The first example serves as a comparison between different, low-order MMRP models of $\mathrm{ON} / \mathrm{OFF}$ streams. Specifically, multiplexing analysis figures are presented for a homogeneous traffic mix of ON/OFF streams, each modelled as a two-state MMRP (exponential OFF, exponential ON, (i) in fig. 3), three-state MMRP (all the combinations, one state being hypo- or hyper-exponential, the other being exponential) and four-state MMRP (both states being either hypo-exponential or hyper-exponential). Fig. 3(a) gives the state diagram for each model along with its parameter values (i.e. the mean-sojourn time at each state and the transition probabilities), while fig. 3 (b) shows the corresponding bufferoccupancy CPDFs for a mix of 7 such streams with a normalised ON rate equal to $c=0.2$. We can see that all the curves share the same origin (non-empty buffer probability), then diverging for increasing buffer contents. The lower curve corresponds to the case with both phases hypo-exponential, while the top-most one to the case having both phases hyperexponential. The other curves are located in-between. 
(ii)
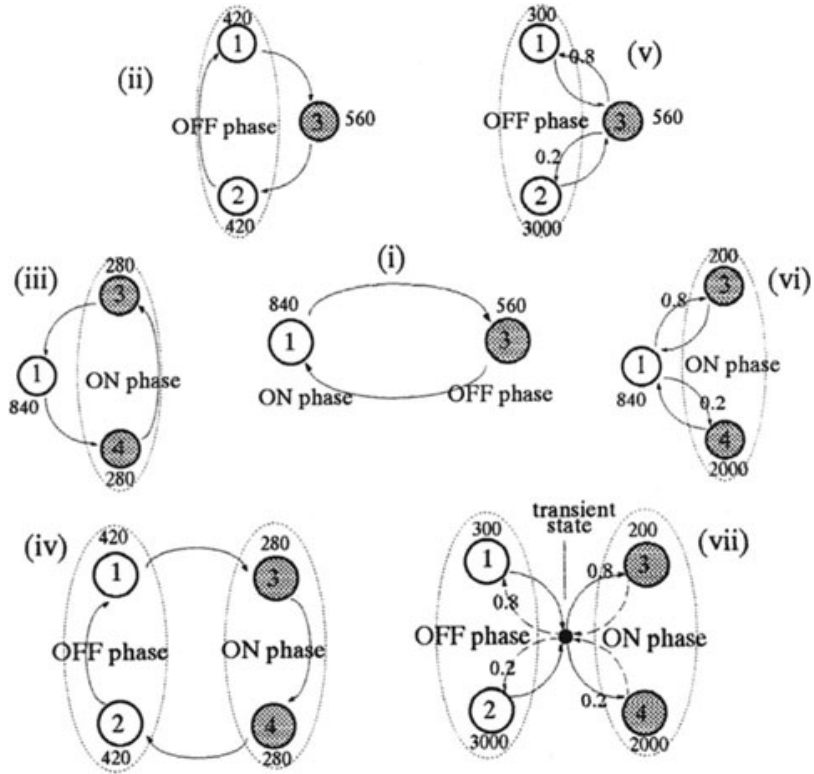

(a)

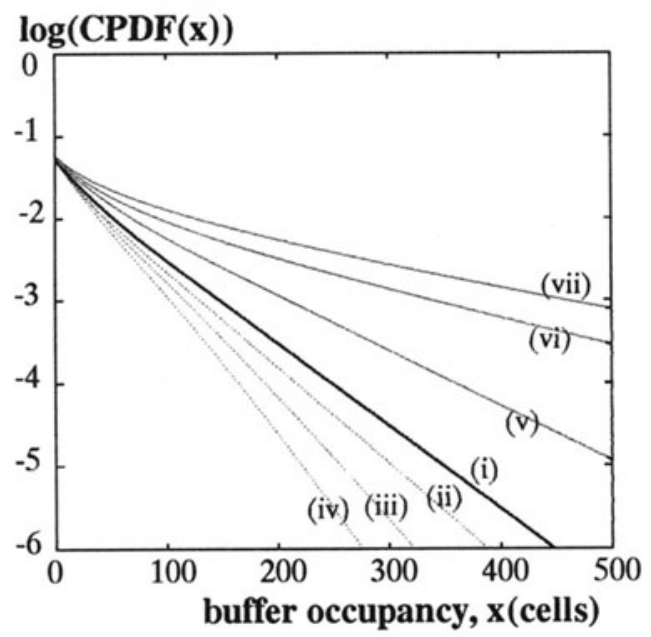

(b)

Figure 3: (a) Low-order MMRP models for ON/OFF traffic streams (b) Buffer occupancy distributions for $N=7$ streams, $c=0.2$ (on the arrows are transition probabilities, on the states are mean sojourn times) 
Although not proven as a general statement, it is observed that hypo-exponential models are more optimistic than the equivalent exponential ones, while the reverse is true for hyper-exponential models. It has also been observed that the distribution of the ON phase is more critical for the multiplexing behaviour than that of the OFF phase (compare curves (ii) with (iii) and (v) with (iv) in fig. 3(b)).

\section{Example 2:}

The second example demonstrates the modelling of a higher-order MMRP (resulting in hyper-exponential $\mathrm{ON}$ and $\mathrm{OFF}$ phases) by using the proposed four-state model and presents the subsequent multiplexing analysis results. In particular, ON/OFF cell streams are generated by shaping data-packet streams, featuring independently distributed packet size (ps) and interpacket distance (ipd), according to a 4-parallel-stages Erlang-type distribution, namely

$$
\begin{aligned}
& \operatorname{Pr}\{x<i p d<x+d x\}=\frac{0.2}{200} e^{-\frac{x}{200}}+\frac{0.4}{800} e^{-\frac{x}{800}}+\frac{0.2}{1600} e^{-\frac{x}{1600}}+\frac{0.2}{3600} e^{-\frac{x}{3600}} \\
& \operatorname{Pr}\{y<p s<y+d y\}=\frac{0.2}{8} e^{-\frac{y}{8}}+\frac{0.4}{32} e^{-\frac{y}{32}}+\frac{0.2}{64} e^{-\frac{y}{64}}+\frac{0.2}{144} e^{-\frac{y}{144}}
\end{aligned}
$$

The shaping rate has been tuned at $20 \%$ of the link rate. Measurements on simulated streams have given the following values for the first three moments of the ON and OFF phases at the output of the shaper:

$$
\begin{aligned}
& t_{1}=407.00, t_{2}=7.37 E+5, t_{3}=2.77 E+9 \\
& s_{1}=1640.00, s_{2}=8.483 E+6, s_{3}=8.19 E+10
\end{aligned}
$$

Now, applying the three-moment-matching approach to modelling of the above streams, as implemented by eq. (15), yields the following parameter values for the four-state model of fig. 1(c):

$$
\begin{aligned}
& \tau_{1}=849.00, \tau_{2}=3568.00, p_{1}=0.71 \\
& \tau_{3}=191.00, \tau_{4}=1345.00, p_{3}=0.81
\end{aligned}
$$

Ten such streams are multiplexed on the same link, as shown in fig. 4(a). The buffer occupancy distribution, as derived by the analysis of section 3 , is shown in fig. 4(b) (curve i), along with the corresponding simulation results (discrete-time cell-level simulation). The same figure displays curves resulting by modelling the multiplexed streams as a three-state MMRP (hyper-exponential ON, exponential OFF for curve ii; and hyper-exponential OFF, 
exponential ON for curve iii) and as a two-state MMRP (exponential ON, exponential OFF for curve iv). One can observe the deviation of the latter from the actual multiplexing performance, while the proposed four-state modelling exhibits a remarkable agreement with the simulation results. In this particular example, the three-state MMRP with a hyperexponential $\mathrm{ON}$ and an exponential OFF phase exhibits a performance close to that of the full, four-state model, thus justifying the respective modelling and multiplexing analysis, as in [16].

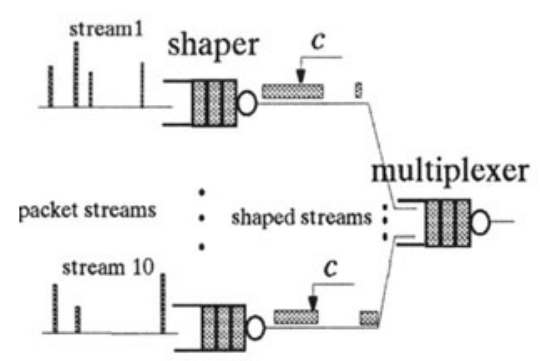

(a)

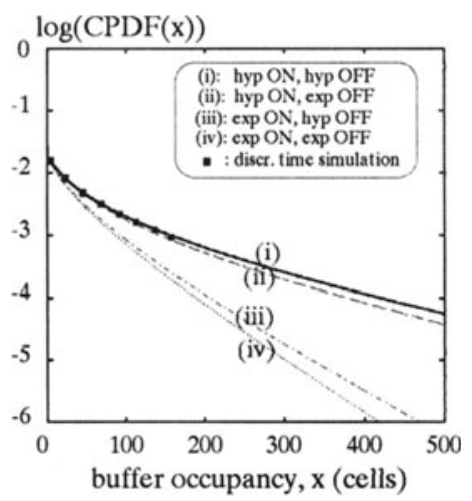

(b)

Figure 4: (a) multiplexing of shaped streams (b) buffer occupancy distributions

\section{Example 3:}

This example is concerned with a real experiment performed on the EXPLOIT ${ }^{2}$ Testbed, a small-scale ATM network built and used for broadband traffic experiments in Europe (for a brief description of the Testbed architecture and related performance evaluation studies see, e.g., [4]). The set-up of the experiment is shown in fig. 5(a): Four connections, the three originating from PCs and the fourth one from a traffic generator producing CBR traffic, are routed through the same multiplexing element (Remote Unit, RUM). The PCs can be programmed to generate ON/OFF traffic in real time, with arbitrary ON and OFF sojourntime distributions and a peak rate up to $55 \mathrm{Mbit} / \mathrm{sec}$, while the constant rate of the CBR connection is also programmable at desired values up to the full link rate $(155.5 \mathrm{Mb} / \mathrm{s})$. At the output of the RUM a test tool is connected which can (among other things) measure the received cells for each connection. Comparison of the number of transmitted cells with the number of those received can give an estimate of the cell losses through the multiplexing element.

Fig. 5(b) shows the expected buffer occupancy distributions as seen by the ON/OFF traffic streams for two-state exponential (curves ii) and four-state hyper-exponential (curves i) ON/OFF traffic and two different CBR loads. These curves provide an upper bound to 
the actual Cell Loss Ratio. The lower the overflow probability the tighter that bound is expected to be. On the same figure we can also see the measured losses, positioned on the actual buffer size of the RUM (28 cells). A quite satisfactory agreement between analysis and experimental results is evidenced. Notice that for the higher load the deviation between experimental and analytical results is larger (as expected), but the relative distance between exponential and hyper-exponential performance is preserved.

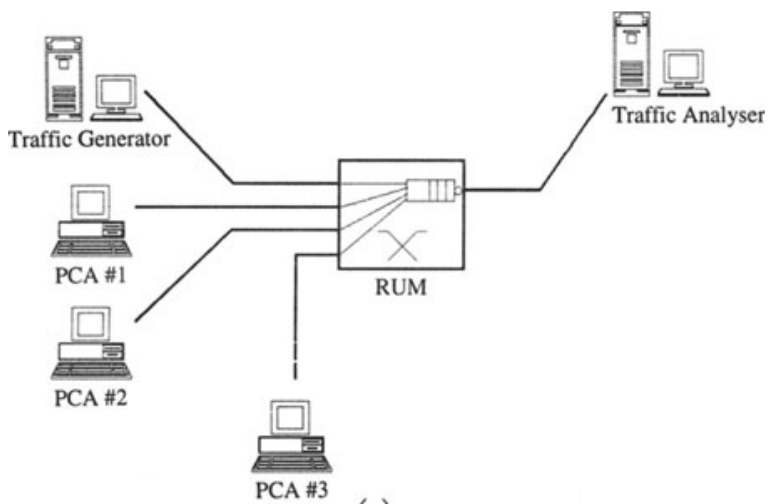

(a)

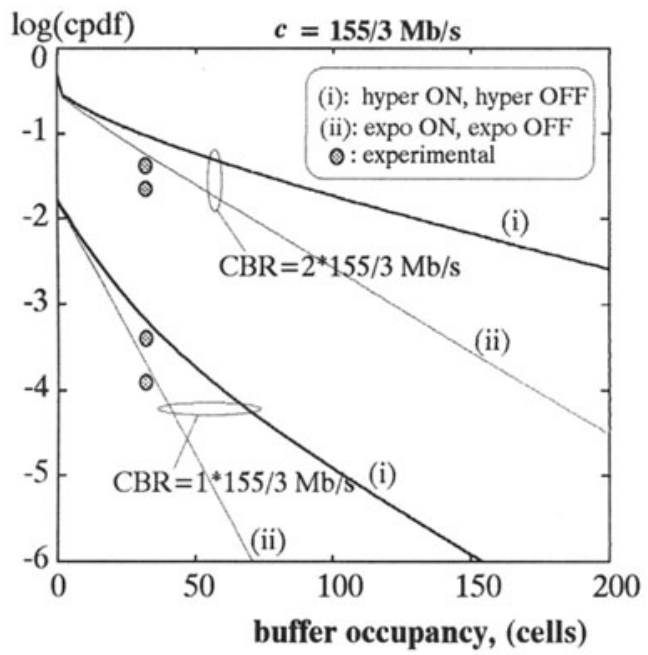

(b)

Figure 5: A real ATM experiment; (a) equipment set-up, (b) expected (-) and measured (우) cell losses 


\section{CONCLUSION}

Traffic streams of the ON/OFF type are expected to dominate in the forthcoming ATM networks. Compared to multirate, the ON/OFF traffic is easier to control, yet offering a statistical multiplexing gain. Multirate streams can be transformed to ON/OFF through a shaping process at the transmitter.

Although a two-state MMRP (an ON-OFF rate process with exponentially distributed $\mathrm{ON}$ and $\mathrm{OFF}$ sojourn times) has attracted much attention in the literature due to its analytical tractability, it fails to serve as a general ON/OFF traffic model with an acceptable accuracy, since hyper-exponential streams (i.e. streams with hyper-exponentially distributed $\mathrm{ON}$ or/and $\mathrm{OFF}$ sojourn times) exhibit a worse performance than their exponential counterparts. Exponential traffic will only be an exception.

A four-state MMRP is proposed in this paper as a means of obtaining a sufficiently accurate, yet computationally tractable analytical model for ON/OFF traffic streams. Under a time-reversibility assumption (which is reasonable for many practical situations of interest) the parameters of the model can be easily assessed by measurements gathered from the actual streams. Furthermore, the multiplexing performance of the proposed model is obtained analytically by using a well-established factorization method. Application examples are given, which demonstrate the applicability of the proposed modelling technique and compare the behaviour of the model to other, lower-order ones.

\section{REFERENCES}

[1] D. Anick, D. Mitra and M.M. Sondhi, "Stochastic theory of a data-handling system with multiple sources", The Bell System Technical Journal, vol. 61, no. 8, October 1982, pp. 1871-1894.

[2] A. Baiocchi, N. Blefari-Melazzi, A. Roverti, and F. Salvatore, "Stochastic Fluid Analysis of an ATM Multiplexer Loaded with Heterogeneous ON-OFF Sources: an Effective Computational Approach," In Proc. INFOCOM '92, pp. 3C.3.1-3C.3.10, 1992.

[3] P.J. Courtois, Decomposability: Queueing and Computer System Applications, Academic Press, New York, 1977.

[4] J. Domingo et.al. "Switching Block Studies, Network Performance Evaluation and Traffic Engineering for ATM," to appear in the European Transactions on Telecommunications, 1994.

[5] A. Elwalid, D. Mitra and T.E Stern, "A Theory of Statistical Multiplexing of Markovian Sources: Spectral Expansions and Algorithms", 1st International Conference on Numerical Solution of MCs, Raleigh 1990

[6] A. Elwalid and D. Mitra, "Effective bandwidth of general Markovian sources and admission control of high speed networks," IEEE/ACM Transactions on Networking, June 1993, pp. 329-343.

[7] R. Guerin, H. Ahmadi and M. Naghshineh, "Equivalent capacity and its application to bandwidth allocation in high-speed networks," IEEE JSAC, vol. 9, pp. 968-981, 1991.

[8] K.P. Kontovasilis and N.M. Mitrou, "Bursty Traffic Modeling and Efficient Analysis Algorithms via Fluid-Flow Models for ATM IBCN," Annals of Operations Research, Vol. 49, special issue on Methodologies for High Speed Networks (1994). 
[9] K. Kontovasilis and N. Mitrou, "Markov Modulated Traffic with Near Complete Decomposability Characteristics and Associated Fluid Queueing Models," to appear in the Applied Probability Journal (Dec. 1995).

[10] K.P. Kontovasilis and N.M. Mitrou, "Stochastic fluid models for a buffer loaded by Markov modulated traffic with near complete decomposability characteristics", Proceedings of the Fifth International Conference on Data Communication Systems and their Performance, Research Triangle Park, North Carolina, Oct. 25-28, 1993.

[11] L. Kosten, "Stochastic theory of data-handling systems with groups of multiple sources," in Performance of Computer Communication Systems, H. Rudin and W. Bux, Eds. Amsterdam, The Netherlands: Elsevier, 1984, pp. 321-331.

[12] B. Maglaris et.al. "Performance models of statistical multiplexing in packet video communications," IEEE Trans. Commun., vol. 20, pp. 834-843, 1988.

[13] D. Mitra, "Stochastic theory of a fluid model of producers and consumers coupled by a buffer, Adv. Appl. Prob. , Vol. 20, 1988, pp. 646-676.

[14] N.M. Mitrou and A. Koukos, "An effective-rate enforcement algorithm for ATM traffic and its hardware implementation", Proceedings of the IBCN\&S, Copenhagen, April 20-23, 1993.

[15] N.M. Mitrou and K.P. Kontovasilis, "An efficient descriptor of the multiplexing attributes of streams", Proceedings of the 4th International Conference on Advances in Communication \& Control, Rhodes, Greece, June 14-18, 1993.

[16] N.M. Mitrou, S. Vamvakos, K. Kontovasilis, "Modelling, Parameter Assessment and Multiplexing Analysis of Burtsy Sources with Hype-exponentially Distributed Bursts," to appear in the Computer Networks and ISDN Systems, 1995.

[17]. RACE Project R1022, "Final Traffic Models and Applications", Deliverable 22/TG03/WG04/DS/R/124/03, Dec. 1991.

[18] J.W. Roberts, "Variable-bit-rate traffic control in B-ISDN", IEEE Communications Magazine, vol. 29, no. 9, September 1991, pp. 50-56.

[19]. T.E. Stern and A.I. Elwalid, "Analysis of separable Markov-modulated rate models for information-handling systems", Advances in Applied Probability, vol. 23, 1991, pp. 105-139.

[20] Y. Xiong, H. Bruneel and G. Petit, "Performance study of an ATM self-routing multistage switch with bursty traffic: simulation and analytic approximation", European Transactions on Telecommunications and Related Technologies (ETT), vol. 4, no. 4, JulyAugust 1993, pp. 443-453.

\section{Appendix A.}

Assertion: The infinitesimal generator in (1) with $0<p_{j}<1, j=1, \ldots, 4$ is time reversible iff (7) holds.

\section{Proof:}

(a) Necessity: Let the steady state probabilities be $\pi_{i}, i=1, . ., 4$. The infinitesimal generator $M$ is time-reversible if and only if it holds 


$$
\pi_{i} M_{i j}=\pi_{j} M_{j i}, \forall i, j
$$

where the relation is non-trivial only for $i \neq j$.

By denoting $\zeta_{i} \equiv \pi_{i} \tau_{i}^{-1}, i=1, . ., 4$, (A.1) is equivalent to the set of equations

$$
\begin{aligned}
& \zeta_{1} p_{1}=\zeta_{3} p_{3} \\
& \zeta_{1}\left(1-p_{1}\right)=\zeta_{4}\left(1-p_{4}\right) \\
& \zeta_{2}\left(1-p_{2}\right)=\zeta_{3}\left(1-p_{3}\right) \\
& \zeta_{2} p_{2}=\zeta_{4} p_{4}
\end{aligned}
$$

By dividing the third equation by the first and, similarly, the fourth by the second we arrive at

$$
\frac{\zeta_{2}}{\zeta_{1}} \frac{1-p_{2}}{p_{1}}=\frac{1-p_{3}}{p_{3}}, \quad \frac{\zeta_{2}}{\zeta_{1}} \frac{p_{2}}{1-p_{1}}=\frac{p_{4}}{1-p_{4}}
$$

and by solving these two for $\frac{\zeta_{2}}{\zeta_{1}}$ and equating the right hand sides, after rearrangement we arrive at (7).

(b) Sufficiency: Let (7) hold and let

$$
\begin{aligned}
\zeta_{1} & =C \\
\zeta_{2} & =\frac{1-p_{3}}{p_{3}} \frac{p_{1}}{1-p_{2}} C \\
\zeta_{3} & =\frac{p_{1}}{p_{3}} C \\
\zeta_{4} & =\frac{1-p_{1}}{1-p_{4}} C
\end{aligned}
$$

where $C$ is a positive normalization constant such that $\sum_{i=1}^{4} \zeta_{i} \tau_{i}=1$. It can be easily verified that the vector $\vec{x}=\left(\zeta_{1} \tau_{1}, \ldots, \zeta_{4} \tau_{4}\right)$ is positive and, given that (7) holds, satisfies $\vec{x} M=\overrightarrow{0}$, with $M$ as in (1). Thus, by the irreducibility of $M, \vec{x}$ is the unique steady state probability vector. Furthermore, given that (7) holds it can be easily seen that the quantities $\zeta_{i}$ satisfy the equations (A.2), thus $\pi_{i} M_{i j}=\pi_{j} M_{j i}, \forall i, j$, proving that $M$ is time reversible. 


\section{Appendix B.}

Consider the model of fig. 1(a) and define:

$$
\lambda_{j}=\tau_{j}^{-1}, \quad \lambda_{i j}=\lambda_{i} \lambda_{j}, \quad \lambda_{i j k}=\lambda_{i} \lambda_{j} \lambda_{k}, \quad i, j, k=1,2,3,4 .
$$

$$
\begin{array}{ll}
\Lambda_{12} & =\lambda_{12}, \quad \Lambda_{13}=\left(1-p_{1} p_{3}\right) \lambda_{13}, \quad \Lambda_{14}=\left[1-\left(1-p_{1}\right)\left(1-p_{4}\right)\right] \lambda_{14}, \\
\Lambda_{23} & =\left[1-\left(1-p_{2}\right)\left(1-p_{3}\right)\right] \lambda_{23}, \quad \Lambda_{24}\left[1-p_{2} p_{4}\right] \lambda_{24}, \Lambda_{34}=\lambda_{34} \\
\Lambda_{123} & =\left[1-p_{1} p_{3}-\left(1-p_{2}\right)\left(1-p_{3}\right)\right] \lambda_{123}, \quad \Lambda_{124}=\left[1-\left(1-p_{1}\right)\left(1-p_{4}\right)-p_{2} p_{4}\right] \lambda_{124} \\
\Lambda_{134} & =\left[1-p_{1} p_{3}-\left(1-p_{1}\right)\left(1-p_{4}\right)\right] \lambda_{134}, \quad \Lambda_{234}=\left[1-\left(1-p_{2}\right)\left(1-p_{3}\right)-p_{2} p_{4}\right] \lambda_{234}
\end{array}
$$

The eigenvalues of $A^{(n)}(z)$ in (9) satisfy the equation $\left|R^{(n)}-M^{(n)} / z-g I\right|=0$, where $I$ is the fourth-order identity matrix. The above equation is written as

$$
g^{4}+\beta_{1} g^{3}+\beta_{2} g^{2}+\beta_{3} g+\beta_{4}=0
$$

where

$$
\begin{aligned}
& \beta_{1}=-\left(2 c+z^{-1} \sum_{j} \lambda_{j}\right) \\
& \beta_{2}=\left\{c^{2}+c z^{-1}\left[2\left(\lambda_{1}+\lambda_{2}\right)+\lambda_{3}+\lambda_{4}\right]+z^{-2} \sum_{\substack{i, j \\
i \neq j}} \Lambda_{i j}\right\} \\
& \beta_{3}=-\left\{c^{2} z^{-1}\left(\lambda_{1}+\lambda_{2}\right)+c z^{-2}\left[\lambda_{12}-\lambda_{34}+\sum_{\substack{i, j \\
i \neq j}} \Lambda_{i j}\right]+z^{-3} \sum_{\substack{i, j, k \\
i \neq j \neq k}} \Lambda_{i j k}\right\} \\
& \beta_{4}=c^{2} z^{-2} \lambda_{12}+c z^{-3}\left(\Lambda_{123}+\Lambda_{124}\right)
\end{aligned}
$$

(the superscript $(\mathrm{n})$ has been omitted for simplicity).

The solution of the above fourth-order polynomial equation can be found in closed form in standard mathematics text books. For the sake of completeness, we quote it here:

By making the transformation $g=y-\beta_{1} / 4$, equation (B.1) is transformed to

$$
y^{4}+\gamma_{1} y^{2}+\gamma_{2} y+\gamma_{3}=0
$$

Then, the solutions of (B.2) are given by 


$$
y_{1,2,3,4}= \pm \sqrt{x_{1}} \pm \sqrt{x_{2}} \pm \sqrt{x_{3}}
$$

where $x_{1}, x_{2}, x_{3}$ satisfy the third-order equation

$$
x^{3}+\frac{\gamma_{1}}{2} x^{2}+\frac{\gamma_{1}^{2}-4 \gamma_{3}}{16} x-\frac{\gamma_{2}^{2}}{64}=0
$$

and the signs in (B.3) are adjusted so that $\left( \pm \sqrt{x_{1}}\right)\left( \pm \sqrt{x_{2}}\right)\left( \pm \sqrt{x_{3}}\right)=-\frac{\gamma_{2}}{8} \quad(4$ valid combinations).

If the time-reversibility assumtion (7) holds, all three roots of (B.4) must be real and positive, given in that case as

$$
x_{j}=2 \sqrt{-Q} \cos \left(\frac{q+2(j-1) \pi}{3}\right)-\frac{\delta_{1}}{3}, \quad j=1,2,3
$$

where $\quad q=\cos ^{-1}\left(\frac{P}{\sqrt{-Q^{3}}}\right), \quad Q=\frac{3 \delta_{2}-\delta_{1}^{2}}{9}, \quad P=\frac{9 \delta_{1} \delta_{2}-27 \delta_{3}-2 \delta_{1}^{2}}{54}$,

and

$$
\delta_{1}=\frac{\gamma_{1}}{2}, \quad \delta_{2}=\frac{\gamma_{1}^{2}-4 \gamma_{3}}{16}, \quad \delta_{3}=-\frac{\gamma_{2}^{2}}{64}
$$

Finally the sought $g$ functions are given as $g(j ; z)=y_{j}-\beta_{1} / 4$, with $y_{j}$ as in (B.3).

It is also easy to compute the first derivative of the $g$ functions which are required by the Newton-Raphson method to solve (13), by differentiating (B.1):

$$
\begin{aligned}
& g^{\prime}=-\frac{\beta_{1}^{\prime} g^{3}+\beta_{2}^{\prime} g^{2}+\beta_{3}^{\prime} g+\beta_{4}^{\prime}}{4 g^{3}+3 \beta_{1} g^{2}+2 \beta_{2} g+\beta_{3}}, \\
& g^{\prime} \equiv \frac{d}{d z} g(j ; z), \quad \beta_{j}^{\prime} \equiv \frac{d}{d z} \beta_{j}, \quad j=1,2,3,4
\end{aligned}
$$

where the derivatives $\beta_{j}^{\cdot}$ can be computed by direct differentiation of (B1.b).

Suppose now that we have a reduced, three-state model, with $M$ and $R$ given by (17). The three $g$ functions, eigenvalues of the corresponding matrix $A(z) \equiv R-M / z$, are roots of the polynomial equation (18), given by (B.5a) and (B.5b), where 


$$
\begin{aligned}
& \delta_{1}=c-z^{-1}\left(\lambda_{1}+\lambda_{3}+\lambda_{4}\right) \\
& \delta_{2}=-\left\{c z^{-1}\left(\lambda_{3}+\lambda_{4}\right)-z^{-2}\left[\lambda_{3} \lambda_{4}+\left(1-p_{1}\right) \lambda_{1} \lambda_{3}+p_{1} \lambda_{1} \lambda_{4}\right] .\right. \\
& \delta_{3}=c z^{-2} \lambda_{3} \lambda_{4}
\end{aligned}
$$

These $g$ functions are real, since the model at hand is time-reversible.

\section{Appendix C}

Assertion: Given three positive numbers $s_{1}, s_{2}, s_{3}$, the inequalities

$$
s_{2}>2 s_{1}^{2}, \quad s_{3}>\frac{3 s_{2}^{2}}{2 s_{1}}
$$

are necessary and sufficient for the $s_{1}, s_{2}, s_{3}$ to represent the first three moments about the origin of a hyper-exponentially distributed, positive random variable, $s>0$, with a p.d.f. given by

$$
\operatorname{Pr}\{x \leq s<x+d x\}=\frac{p_{1}}{\tau_{1}} e^{-\frac{x}{\tau_{1}}}+\frac{1-p_{1}}{\tau_{2}} e^{-\frac{x}{\tau_{2}}}, \quad 0<p_{1}<1,\left(\tau_{1} \neq \tau_{2}\right)>0,
$$

\section{Proof:}

(a) Necessity: Suppose that the p.d.f. of the r.v. $s$ is given by (C.2). Then

$$
\begin{gathered}
s_{1} \equiv E\{s\}=p_{1} \tau_{1}+\left(1-p_{1}\right) \tau_{2} \\
s_{2} \equiv E\left\{s^{2}\right\}=2\left[p_{1} \tau_{1}^{2}+\left(1-p_{1}\right) \tau_{2}^{2}\right], \\
s_{3} \equiv E\left\{s^{3}\right\}=6\left[p_{1} \tau_{1}^{3}+\left(1-p_{1}\right) \tau_{2}^{3}\right]
\end{gathered}
$$

from which one gets

$$
\begin{aligned}
& s_{2}-2 s_{1}{ }^{2}=2 p_{1}\left(1-p_{1}\right)\left(\tau_{2}-\tau_{1}\right)^{2} \\
& s_{1} s_{3}-3 s_{2}{ }^{2} /\left(2 s_{1}\right)=6 p_{1}\left(1-p_{1}\right) \tau_{1} \tau_{2}\left(\tau_{2}-\tau_{1}\right)^{2}
\end{aligned}
$$

Given that $0<p_{1}<1, \tau_{1}, \tau_{2}>0, \tau_{1} \neq \tau_{2}, \quad$ the right-hand sides of (C.4) are strictly positive. 
(b) Sufficiency: Suppose now that (C.1) holds true for three positive numbers $s_{1}, s_{2}, s_{3}$. Define

$$
\begin{aligned}
& x=\frac{\frac{s_{3}}{6}-\frac{s_{2}}{2} s_{1}}{\frac{s_{2}}{2}-s_{1}{ }^{2}}, \quad y=s_{1} x-\frac{s_{2}}{2} \\
& \tau_{1}=\frac{x-\sqrt{x^{2}-4 y}}{2}, \quad \tau_{2}=\frac{x+\sqrt{x^{2}-4 y}}{2}, \quad p_{1}=\frac{\tau_{2}-s_{1}}{\tau_{2}-\tau_{1}}
\end{aligned}
$$

By virtue of (C.1), both the numerator and the denominator of $x$ in (C.5a) are strictly positive . Also, from (C.5a), $y=s_{1} x-\frac{s_{2}}{2}=\frac{s_{1} s_{3} / 6-s_{2}{ }^{2} / 4}{s_{2} / 2-s_{1}{ }^{2}}>0$. Furthermore, the quantity $x^{2}-4 y$, by substituting $y$ from (C.5a), can be written as $x^{2}-4 s_{1} x+2 s_{2}$, which is positive for any value of $x$, since its discriminant, $4 s_{1}{ }^{2}-2 s_{2}$, is negative, due to (C.1). We therefore deduce that both of the $\tau_{1}$ and $\tau_{2}$ in (C.5b) are real and positive, satisfying the equations $\tau_{1}+\tau_{2}=x, \quad \tau_{1} \tau_{2}=y$. It is also easy to verify that $\tau_{1}<s_{1}<\tau_{2}$, which gives $p_{1} \in(0,1)$.

Equations (C.5a) can be easily transformed to $s_{1} x-y=\frac{s_{2}}{2} \& x \frac{s_{2}}{2}-s_{1} y=\frac{s_{3}}{6}$, which in turn, by using the equation $p_{1}\left(\tau_{1}-\tau_{2}\right)=s_{1}-\tau_{2}$ (from (C.5b)), give:

$$
\left.\begin{array}{l}
p_{1}\left(\tau_{1}-\tau_{2}\right)+\tau_{2}=s_{1} \\
p_{1}\left(\tau_{1}^{2}-\tau_{2}^{2}\right)+\tau_{2}^{2}=\frac{s_{2}}{2} \\
p_{1}\left(\tau_{1}^{3}-\tau_{2}^{3}\right)+\tau_{2}^{3}=\frac{s_{3}}{6}
\end{array}\right\}
$$

In (C.6) we recognise equations (C.3) giving the first three moments of a r.v. with a p.d.f. as in (C.2). This completes the proof. 